\title{
Modeling Pilot Situation Awareness
}

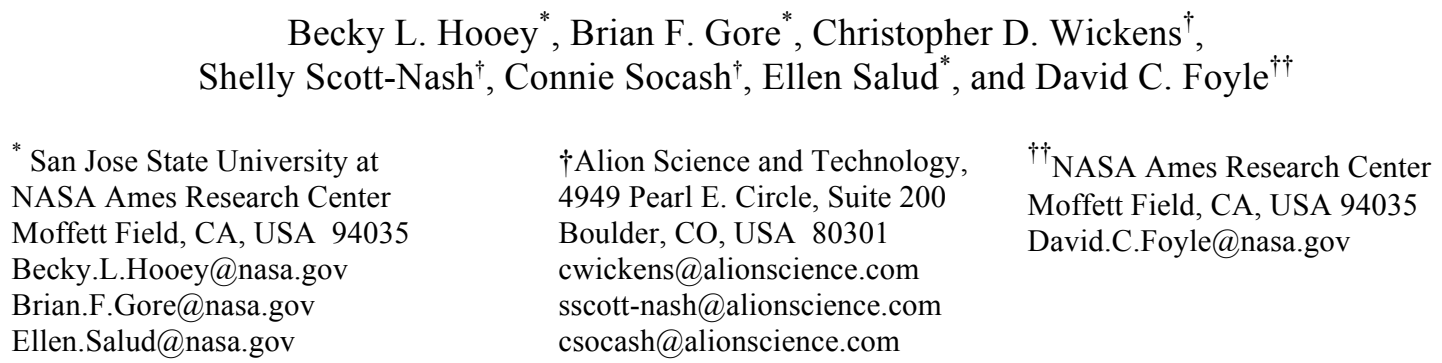

\section{Abstract}

The Man-machine Integration Design and Analysis (MIDAS) human performance model was augmented to improve predictions of multi-operator situation awareness (SA). In MIDAS, the environment is defined by situation elements (SE) that are processed by the modeled operator via a series of sub-models including visual attention, perception, and memory. Collectively, these sub-models represent the situation assessment process and determine which SEs are attended to and comprehended by the modeled operator. SA is computed as a ratio of the Actual SA (the number of SEs that are detected or comprehended) relative to the Optimal SA (those deemed required or desired for the operator to complete his/her task).

A high-fidelity application model of a two-pilot commercial crew during the approach phase of flight was generated to demonstrate and verify the SA model. Two flight deck display configurations, hypothesized to support pilot SA at differing levels, were modeled. The results presented include the ratio of actual to optimal SA for three high-level tasks: Aviate, Separate, and Navigate. The model results verified that the SA model operates as expected and is sensitive to scenario characteristics including display configuration and pilot responsibilities.

\section{Introduction}

In the Next Generation (NextGen; JPDO, 2009) of aviation operations it is anticipated that there will be substantially more information available to pilots on the flight deck (e.g., weather, wake, terrain, traffic trajectory projections) to support more precise and closely coordinated operations. Safe and efficient task performance within complex sociotechnical systems depends on operators acquiring and maintaining appropriate levels of situation awareness (SA; Salmon, Stanton, Walker \& Jenkins, 2009), and as such, a critical issue is how well the flight deck will support the pilots' ability to acquire and maintain SA of relevant information in the NextGen environment.

Evaluating pilot SA using human-in-the-loop (HITL) simulations requires sufficient understanding of the operating environment, technologies, and automation to enable the development of functional prototypes. However, many NextGen concepts have not yet reached this level of maturity. Human performance models (HPMs) on the other hand, allow researchers to evaluate new aviation displays, automation, operations, and procedures in order to identify candidate concepts that are likely to increase safety and efficiency and to weed out those that have the potential for problems, before significant development costs have been incurred (Hooey \& Foyle, 2008). As such, HPMs present an excellent test bed to evaluate the impact of NextGen concepts and technologies on pilot SA.

Many definitions of SA can be found in the literature, however, the most commonly accepted definition is that offered by Endsley (1995) who defined SA as the perception of the elements in the environment within a volume of time and space, the comprehension of their meaning, and the projection of their status 
in the near future. SA is dynamic, in that it can be updated or diminished as a situation evolves, as a result of ongoing bottom-up/top-down information processing (or situation assessment).

To date, efforts to computationally model and predict SA have been few (for examples, see Zacharias, Miao, Illgen, Yara, \& Siouris, 1995; Wickens et al., 2008; Jones et al., 2010). Furthermore, for the design of flight deck displays and procedures for two-pilot crews, it is necessary to consider not only individual SA, but also how SA is distributed across the crew members (Salmon, Stanton, Walker \& Jenkins, 2009). Distributed SA (DSA) is differentiated from models of Team SA that focus on a shared understanding of the same situation (Bolstad, Cuevas, Costello, \& Rousey, 2005; Bolstad \& Endsley, 2000; Nofi, 2000), and from models of Shared SA that focus on the degree of overlap in common SA elements between team members (Endsley \& Jones, 1997). In contrast, distributed SA (sometimes referred to as systemic SA or compatible SA) is applied to joint cognitive systems in which each operator holds a different view of the situation that is compatible, but not redundant, with other operators within the system (Salmon, Stanton, Walker \& Jenkins, 2009). In this manner, each operator's SA forms a part of the distributed SA of the entire system and is required collectively for the entire system to work. The concept of DSA is applicable to commercial aviation operations. Especially in light of the increased availability of information and multiple data sources anticipated in the NextGen flight deck, it is likely that the flight deck will shift from an environment in which both pilots share equal responsibilities for all facets of flight, to one in which the two pilots each specialize in different aspects of data management and system monitoring. The question then becomes how to design displays and procedures to support optimal distribution of SA.

This paper describes recent enhancements to the Man-machine Integration Design and Analysis System (MIDAS) that enable improved predictions of pilot SA. First, a brief description of the MIDAS human performance modeling tool will be provided, followed by a description of the specific MIDAS augmentations made to model both the situation assessment process and the situation awareness product. Next, results of an application model that predicts SA of a two-pilot flight crew will be presented to verify that the model is sensitive to changes in display configurations and pilot responsibilities.

\subsection{Man-machine Integration Design and Analysis System (MIDAS)}

MIDAS is a 3-D rapid prototyping human performance modeling and simulation environment that facilitates the design, visualization, and computational evaluation of complex man-machine system concepts in simulated operational environments (see Gore, 2010, this volume; Gore \& Smith, 2006; Gore, Hooey, Wickens, \& Scott-Nash, 2008). MIDAS links a virtual human, comprised of a physical anthropometric character, to a computational cognitive structure that represents human capabilities and limitations. The cognitive component includes perceptual (visual and auditory), visual attention, and memory mechanisms. Information flows from the environment to the perceptual and cognitive submodels, then to a task network representation of the procedures, that then feeds back into the environment. Actions carried out by the modeled operator impact the performance of the model in a closed-loop fashion. The complex interplay among bottom-up and top-down processes enables the emergence of unforeseen and non-programmed behaviors.

\section{$2 \quad$ Modeling Situation Assessment and Situation Awareness}

The MIDAS SA model was first developed by Shively, Brickner, and Silbiger (1997) and has recently been augmented to enable improved predictions of multi-operator SA in NextGen aviation concepts. In MIDAS, the situation context defines what information is important to the modeled operator in the situation (Shively, Brickner, \& Silbiger, 1997). The model adopts Pew's (1995) definition that "a situation is a set of environmental conditions and system states with which the participant is interacting that can be characterized uniquely by a set of information, knowledge, and response options". At a minimum, in modeling NextGen applications, the context is defined by the phase of flight (taxi, 
departure, en route, approach, or land), but may be broken down to finer levels of granularity, or along other dimensions such as nominal and off-nominal (Hooey et al., 2009) operations. For each context, the operators' high-level tasks are defined. For NextGen aviation models, the default high-level tasks adhere to the following hierarchy of task importance ${ }^{1}$ : Aviate, Separate, Navigate, Communicate, and Systems Management (see Schutte \& Trujillo, 1996). For SA, these can be subdivided; for example, the task of Separate can be divided into "Separate from traffic" and "Separate from terrain". The importance of each task is defined (as high, medium, or low) for each operator and each context.

Within each context, the environment is broken down into 'Situational Elements' (SEs), which are pieces of information that are necessary to support the operator's high-level tasks (Shively, Brickner \& Silbiger, 1997). Subject matter experts define the SEs that are required or desired to complete each high-level task. For example, for the task of 'Aviate', the SE 'altitude' is required, but the SE 'angle of attack' (which is a display that presents the angle of the wing relative to the wind and warns of stall conditions) is desired. Although angle of attack supports pilot performance and makes the task easier, it is not strictly necessary, or required. The accessibility of each SE is defined by the analyst using a set of design heuristics including: display modality (visual, auditory), legibility (size, contrast), permanence (always visible, automatically presented, requires key strokes), and format (text, graphical). For example, spatial information that is conveyed by a text display would be classified as less accessible than information that is conveyed graphically. Similarly, information that is buried within a menu structure would be classified as being less accessible than information that does not require a button press to access.

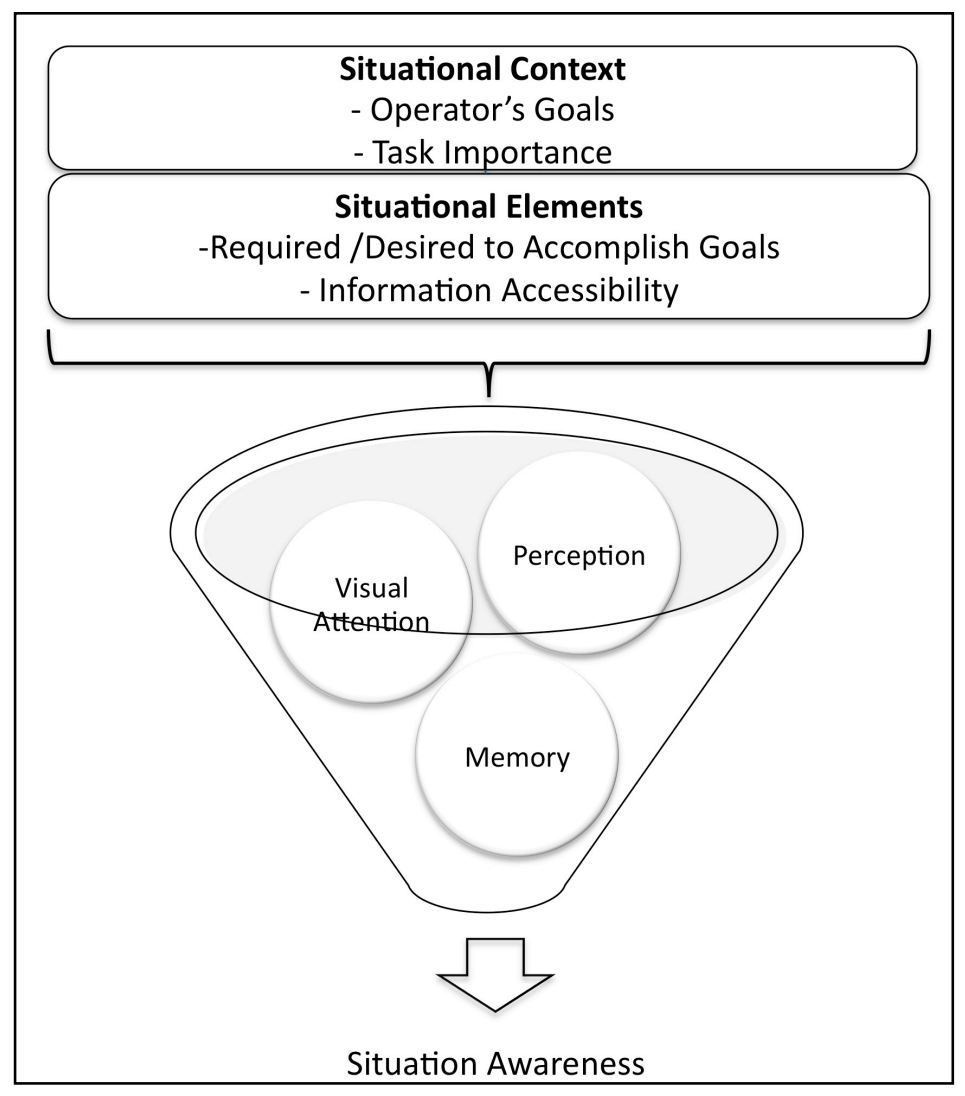

Figure 1. Situation Awareness model in MIDAS

\footnotetext{
1 Schutte \& Trujillo (1996) define a four-level workload management hierarchy: Aviate, Navigate, Communicate, and Systems management. Separate is added here to accommodate new flight-deck responsibilities anticipated in the NextGen environment
} 
Information from the environment flows to the modeled operator and is processed by several sub-models within MIDAS including visual attention, perception, and memory. Together, these sub-models comprise the situation assessment process within MIDAS.

The visual attention sub-model is a previously validated model (Wickens, Goh, Helleberg, Horrey, \& Talleur, 2003; Wickens et al., 2008) that dictates how the pilot's visual scan is driven around the cockpit based on four parameters: Salience, Expectancy, Effort, and Value (SEEV) (See Gore, 2010, this volume, for a discussion of the integration of SEEV into MIDAS). In a top-down fashion, the situation context determines the expectancy and value of any given SE. For example, the expectancy and value of altitude is higher during descent and approach than en-route. The display format and layout determine salience and effort in a bottom-up fashion. Visual attention is drawn to SEs that are salient (e.g., high contrast or flashing alerts) and inhibited by the effort required to visually sample the information (a function of the physical separation of information within the flight deck).

The perception sub-model (see Gore, Hooey, Wickens, \& Scott-Nash, 2009) processes both visual and auditory information, and represents perception as a series of stages that information must pass through in order to be processed. Visual perception in MIDAS depends on three factors - the amount of time the observer dwells on an object, the perceptibility of the observed object, and the accessibility of the information. In the current implementation of MIDAS, perception is a three-stage (undetected, detected, comprehended), time-based perception model for objects inside the workstation (e.g., an aircraft cockpit). The model computes the upper level of detection that can be achieved by the average unaided eye if the observer dwells on it for a requisite amount of time. Once an SE is comprehended, the operator is assumed to have acquired SA of the SE. The perceptibility of each object that falls into the operator's field of view is based on properties of the observed object, the visual angle of the object and environmental factors. One factor that determines perceptibility is the accessibility of the information. An SE with low accessibility requires longer to comprehend, and thus has a corresponding decrement in SA.

Finally, after an SE is comprehended, it is subject to the constraints of the memory sub-model, which degrades SA as a function of time since last accessed. The memory model in MIDAS causes the perception level of a 'comprehended' display to drop to 'detected' after the retrievability threshold of working memory $(5 \mathrm{sec})$ has been surpassed, and perception drops fully to 'undetected' after the retrievability threshold of long-term working memory $(300 \mathrm{sec})$ has been surpassed ${ }^{2}$. As the maximum perception level for an SE drops, there is a corresponding drop in SA.

The information in the environment flows through the situation assessment process and yields a metric of SA for each operator's high-level task (Aviate, Separate, Navigate, Communicate, and Separate). The SA metric in MIDAS computes the ratio of SEs that are detected or comprehended (Actual SA) to the SEs that define the ideal state (Optimal SA). 
Actual SA. For each high-level task ( $i$ ), at time ( $t$ ), Actual SA (See Equation 1) is calculated as the weighted sum of $m$ Required SEs and $n$ Desired SEs multiplied by the perception level $(p)$. Note that if an SE is available on more than one display simultaneously, the highest perception level attained is applied. For SEs within the cockpit, $p$ has values of 0 if the SE is undetected, .5 if detected, and 1.0 if comprehended. Required SEs have a weight of 2 and desired SEs have a weight of 1.

$$
\begin{aligned}
& S A_{\text {Actual }}\left(t_{i}\right)=\sum_{r=1}^{m} 2 \bullet p_{\text {irt }}+\sum_{d=1}^{n} 1 \bullet p_{\text {idt }} \\
& \text { Where } p_{\text {idt }} \text { and } p_{\text {irt }} \text { have values: } 0 \text { for undetected SEs } \\
& 0.5 \text { for detected SEs } \\
& 1.0 \text { for comprehended SEs } \\
& \text { requiredSEs desiredSES }
\end{aligned}
$$

Optimal SA. Optimal SA (see Equation 2) reflects awareness the operator would have if he/she comprehended all the information that is required and desired for the task at any given moment. Therefore for each high-level task $(i)$, at time ( $t$ ), Optimal SA is the weighted sum of $m$ Required SEs and $n$ Desired SEs multiplied by $p$ at any given moment; where $p$ is always equal to 1.0. Required SEs have a weight of 2 and Desired SEs have a weight of 1.

$$
\begin{gathered}
S A_{\text {Optimal }}\left(t_{i}\right)=\sum_{r=1}^{m} 2 \bullet p_{\text {irt }}+\sum_{d=1}^{n} 1 \bullet p_{\text {idt }} \\
\Downarrow \\
\Downarrow \\
\text { required SEs } \quad \text { desired SEs }
\end{gathered}
$$

Where $p_{\text {idt }}$ and $p_{\text {irt }}$ have values of 1.0

SA Ratio (See Equation 3) is the ratio of Actual SA to Optimal SA. It yields a value from 0 (no SA) to 1(maximal SA) that reflects the proportion of SEs that the operator has awareness.

$$
S A_{\text {Ratio }}\left(t_{i}\right)=S A_{\text {Actual }}\left(t_{i}\right) / S A_{\text {Optimal }}\left(t_{i}\right)
$$

\section{Application Scenario}

A high-fidelity model of a two-pilot crew flying an approach into an airport was developed. The model included pilot tasks such as manipulating flight controls, monitoring flight instruments, scanning out the window to maintain separation from traffic and to obtain a visual sighting of the runway, monitoring aircraft system status, and communicating within the cockpit and with ATC. For the purposes of this model, the Captain was assumed to be the pilot flying (left seat) and the First Officer was the pilot-notflying (right seat).

The scenario started with the aircraft at $2200 \mathrm{ft}$ altitude on a normal descent into Dallas/Fort Worth International Airport. The scenario was run with two configurations (Current-day and Augmented) that varied both the flight deck display configuration and pilot responsibilities in a manner expected to impact the time to comprehend information, and, in turn, SA. Each configuration is described next.

Current-day Configuration: The flight deck was equipped with a minimal set of current-day glasscockpit displays including a Primary Flight Display (PFD) that depicted altitude, speed, pitch, bank, and heading and a Navigation Display (ND) that graphically depicted the current flight path via a magenta line. During the approach phase, each pilot's ND was set at a $20 \mathrm{nmi}$ scale. The Captain's ND depicted traffic and terrain whereas the first officer's depicted traffic and weather. Consistent with current day operations, both pilots shared the same hierarchy of importance for the tasks of Aviate, Separate, 
Navigate, Communicate and Systems.

Augmented Configuration: The Captain (left seat) was equipped with a head-up display (HUD) that depicted the primary flight instruments and a highway-in-the-sky (HITS) that was superimposed over the out-the-window view (Fadden, Ververs \& Wickens, 2001) and a ND with settings consistent with the current-day configuration (20 nmi range, showing traffic and terrain). The First Officer was equipped with a current-day PFD and a ND with an advanced 3-D cockpit display of traffic information (CDTI; Granada, Dao, Wong, Johnson \& Battiste, 2005). The First Officer's ND showed predictive information about traffic and weather trajectories allowing for improved visualization of the route relative to the environment. The pilots' responsibilities were set such that each had unique task hierarchies. The Captain's emphasis was placed on the tactical task of Aviate and Separate (from immediate hazards). The First Officer's main responsibility was the strategic planning tasks of Navigate and Separate (from global hazards).

\subsection{Results}

Figure 2 presents SA for both pilots for the Current-day (left panel) and Augmented (right panel) configurations. The figure shows the pilots' awareness of the SEs for the tasks of Aviate, Separate (from hazards), and Navigate (to waypoints). As can be seen, in the Current-day configuration, there were only negligible differences between the Captain and First Officer's SA for each of the three high-level tasks (Aviate, Separate, and Navigate). This was expected, since both pilots shared a similar display configuration and shared equal responsibility for maintaining awareness of all SEs in the environment.

The Augmented configuration demonstrates a different pattern of results, again consistent with expectations. Recall that in the Augmented scenario, it was assumed that the Captain would place highest priority on the tactical tasks of Aviate and Separate from immediate hazards as supported by a HUD with a HITS display. This is clearly reflected in the Captain's SA for the Aviate task, which was higher in the Augmented condition than the Current Day condition. Likewise, in the Augmented condition, the Captain's SA of the Aviate task was higher than that of the First Officer. Further, recall that in the Augmented scenario, the First Officer had an advanced 3-D CDTI that supported strategic Navigate and Separate tasks. This is reflected in the First Officer's increased SA for the tasks of Separate and Navigate, relative to the Captain, in the Augmented configuration.
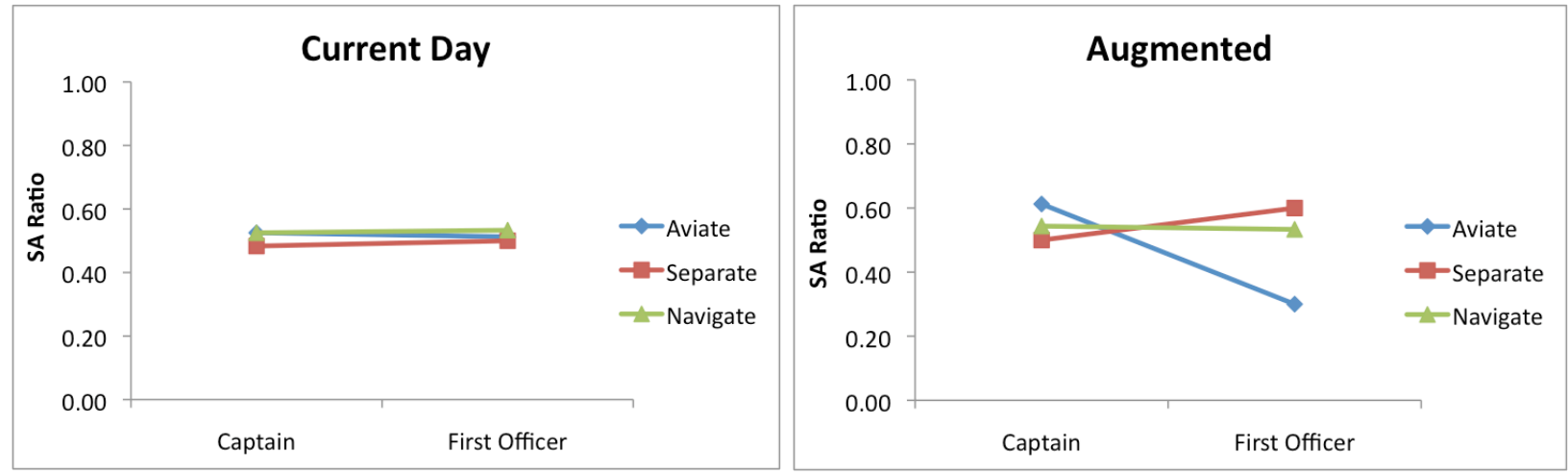

Figure 2. Captain and First Officer SA ratio for the tasks of Aviate, Separate and Navigate as a function of display configuration (Current day, left and Augmented, right). SA ratio is presented on a scale from 0 (no awareness) to 1.0 (maximum awareness) 
Comparing the Current-day to Augmented configurations, it is clear that the distribution of SA has changed in a manner consistent with expectations as a function of the procedural and display manipulations in the Augmented conditions. System-wide, the Augmented configuration enabled a higher level of SA for the task of Aviate (by the Captain) and Separate (by the First Officer) than was attained in the Current-day scenario.

\section{Discussion}

The MIDAS model was augmented yielding improved predictions of multi-operator SA in NextGen aviation operations. Augmentations to the situation assessment processes within MIDAS included the addition of the SEEV model to direct attention within the cockpit as a function of four parameters: Salience, Effort, Expectancy and Value. The SA metric was augmented to allow for the prediction of SA as a function of the operator's high-level tasks (such as shown above, Aviate, Separate, and Navigate). The model also allows for SEs to be characterized according to their level of importance for task completion (required or desired) and for SA to be degraded as a function of information accessibility. It is acknowledged that this model is limited in that it mostly addresses the first of Endsley's (1995) three stages of SA - specifically, the perception of elements in the environment. Future research efforts will be aimed at addressing the subsequent two stages of SA: Comprehension and projection.

The SA model was verified using a high-fidelity simulation model of a two-pilot crew conducting an approach into an airport. Two display configurations were compared: 1) a Current-day display configuration in which pilots were equipped with displays consistent with today's commercial operations and shared a common task hierarchy; and, 2) an Augmented configuration in which the Captain was equipped with a HITS / HUD to support the tactical tasks of Aviate and Separate from local hazards, and the First Officer was equipped with an advanced navigation display to support strategic planning and the task of Navigate. The model output revealed that the SA model was sensitive to differences in display configurations and pilot responsibilities. While future efforts will undertake a formal validation of this model by comparing the model output to human-in-the-loop data, this work represents preliminary steps toward the development of a model-based tool that can be used to predict system-wide, or distributed, SA as a function of procedures and display configurations.

The concept of DSA has only recently emerged in the literature, and empirical research is still required to better understand the construct. The determination of "roles and responsibilities" among all of the human operators (including air traffic controllers and pilots) and emerging automation and technologies, has been identified as one of the primary human factors challenges within the NextGen research community (JPDO, 2009). It is important to better understand environments and situations for which it is better to distribute SA across multiple operators and when it is better that all operators maintain a shared awareness of the same information. With the model described above, it is next possible to evaluate the pilots' ability to detect off-nominal (Hooey et al., 2009) or unexpected events within the cockpit or in the external environment to better determine if the distribution of SA achieved in the Augmented configuration indeed yields improved performance and safety benefits.

\section{References}

Bolstad, C. A., Cuevas, H. M., Costello, A. M., \& Rousey, J. (2005). Improving situation awareness through crosstraining. Proceedings of the $49^{\text {th }}$ Meeting of the Human Factors and Ergonomics Society, Santa Monica, CA: HFES.

Bolstad, C. A., \& Endsley, M. R. (2000). The effect of task load and shared displays on team situation awareness. Proceedings of the 44th Annual Meeting of the Human Factors and Ergonomic Society. Santa Monica, CA: HFES.

Endsley, M. R. (1995). Toward a theory of situation awareness in dynamic systems. Human Factors 37(1), $32-64$. 
Endsley, M. R., \& Jones, D. G. (1997). Situation awareness information dominance and information warfare (Technical Report 97-01). Dayton, OH: Wright-Patterson AFB.

Ericsson, K. A., \& Kintsch, W. (1995). Long-term working memory. Psychological Review, 102(2), $211-245$.

Fadden, S. Ververs, P.M., \& Wickens, C.D. (2001). Pathway HUDs: Are they viable? Human Factors, 43 , 173 193.

Gore, B. F. (2010, this volume). Man-machine Integration Design and Analysis System (MIDAS) v5: Augmentations, Motivations, and Directions for Aeronautics Applications. Proceedings of the Human Modelling of Assistive Technologies Workshop. Begirate, Italy.

Gore, B.F. \& Smith, J.D. (2006). Risk assessment and human performance modeling: The need for an integrated approach. International Journal of Human Factors of Modeling and Simulation, 1(1), 119-139.

Gore, B. F., Hooey, B. L., Wickens, C. D., \& Scott-Nash, S. (2009). A computational implementation of a human attention guiding mechanism in MIDAS v5. In. V.G. Duffy (Ed.). Digital Human Modeling, HCII (pp. 237246). Heidelberg: Springer-Verlag.

Granada, S., Dao, A. Q., Wong, W., Johnson, W. W., \& Battiste, V. (2005). Development and integration of a human-centered volumetric cockpit display for distributed air-ground operations. Proceedings of the $13^{\text {th }}$ International Symposium on Aviation Psychology. Oklahoma City, OK.

Hooey, B. L., \& Foyle, D. C. (2008). Advancing the state of the art of human performance models to improve aviation safety. In D. C. Foyle, \& B. L. Hooey (Eds.), Human Performance Modeling in Aviation. Boca Raton: CRC Press/Taylor \& Francis.

Hooey, B. L., Wickens, C. D., Salud, E., Sebok, A., Hutchins, S., \& Gore, B. F. (2009). Predicting the unpredictable: Estimating human performance parameters for off-nominal events. Proceedings of the 15th International Symposium on Aviation Psychology. Dayton, $\mathrm{OH}$ : Wright State University.

Joint Planning and Development Office (2009). Concept of Operations for the Next Generation Air Transport System, v3.0 (October, 2009). http://www.jpdo.gov/library/NextGen_ConOps_v3\%200.pdf

Jones, R. E. T., Connors, E. S., Mossey, M. E., Hyatt, J. R., Hansen, N. J., \& Endsley, M. R., (2010). Modeling situation awareness for Army infantry platoon leaders using fuzzy cognitive mapping techniques. Proceedings of the Behavior Representation in Modeling and Simulation (BRIMS) Conference. Charleston, SC.

Miller, G. A. (1956). The magical number seven, plus or minus two: Some limits on our capacity for processing information. Psychological Review, 63(2), 81-97.

Nofi, A. (2000). Defining and measuring shared SA (CRM D0002895.A1/Final). Alexandria, Virginia: Center for Naval Analyses.

Pew, R. W. (1995). The state of situation awareness measurement: circa 1995. In D. Garland \& M. Endsley (Eds.). Experimental Analysis and Measurement of Situation Awareness, 7-16, Daytona Beach, FL: Embry-Riddle.

Salmon, P. M., Stanton, N. A., Walker, G. H., \& Jenkins, D. P. (2009). Distributed Situation Awareness. Burlington, Vermont: Ashgate.

Schutte. P. C., \& Trujillo, A. C. (1996). Flight crew task management in non-normal situations. Proceedings of the $40^{\text {th }}$ Annual Meeting of the Human Factors and Ergonomics Society, 244-248, Santa Monica, CA: HFES.

Shively, R. J., Brickner, M., \& Silbiger, J. (1997). A computational model of situational awareness instantiated in MIDAS. Proceedings of the Ninth International Symposium on Aviation Psychology, Columbus, Ohio.

Wickens, C. D., Goh, J., Helleberg, J., Horrey, W. J., \& Talleur, D. A. (2003). Attentional models of multitask pilot performance using advanced display technology. Human Factors, 45(3), 360-80.

Wickens, C. D. \& McCarley, J., Alexander, A., Thomas, L. C., Ambinder, M., \& Zheng, S. (2008). In D. C. Foyle, \& B. L. Hooey (Eds.), Human Performance Modeling in Aviation. Boca Raton: CRC Press/Taylor \& Francis.

Zacharias, G. L., Miao, A. X., Illgen, C., Yara, J. M., \& Siouris, G. M. (1995). SAMPLE: Situation awareness model for pilot in-the-loop evaluation. First Annual Conference on Situation Awareness in the Tactical Air Environment. Patuxent River, MD: Naval Air Warfare Center

\section{Acknowledgment}

This research was funded by the National Aeronautics and Space Administration (NASA) Aviation Safety (AvSAFE) Program (Integrated Intelligent Flight Deck Technologies (IIFTD) / System Design \& Analysis (SDA) project). 